\title{
UN ESSAI SUR LA PERSONNE EN DIACHRONIE : COMMENT LA PERTE DE LA FLEXION VERBALE CHANGE LE POINT DE VUE SUR LA PERSONNE
}

\section{INTRODUCTION}

On peut envisager la notion de personne sous de nombreux points de vue : philosophique, sociologique, économique, linguistique, juridique... Les expressions telles que PC (personal computer), voiture personnelle, opinion personnelle, personne morale etc. témoignent de son omniprésence dans différents domaines de la vie humaine. En linguistique on ne manque pas de termes liés à la personne : pronoms personnels, $i l$ im/unipersonnel, la personne omniprésente, sphère personnelle et autres.

Il n'est presque pas nécessaire de discuter de l'importance de la personne en linguistique. Il suffit de se rappeler le fameux tableau de la prédicativité de Moignet (1981 : 26) et de voir que « le pilier central de l'édifice de la prédicativité » (Ibid. 16) qui est le substantif contient obligatoirement la catégorie de la personne. Ainsi, selon Moignet, « la personne est le support de tout l'édifice formel de cette partie de la langue (du substantif, T. F.)» (Ibid. : 17).

Tandis que pour Moignet, le substantif occupe la place centrale de tout système linguistique, pour Tesnière (1988 [1959]), c'est le verbe. Mais leurs positions ne sont pas incompatibles. Dans la phrase Alfred parle, Tesnière ne voit pas deux éléments (le nom et le verbe) mais plutôt trois éléments : il introduit le terme de connexion qui sert de liaison entre le nom et le verbe. Cette connexion n'est autre que la personne qui se trouve dans le nom et dans le verbe en même temps. Si on change le rang de la personne dans un de ces deux éléments, il faut le changer aussi dans l'autre élément. Ainsi *Alfred parlons. * Alfred et Pierre parle., ne sont pas des phrases attestées.

De ces exemples on peut conclure que ni le nom ni le verbe ne peut se trouver seul au centre du système langagier. Il leur faut la catégorie de la personne.

En ce qui concerne la catégorie de la personne en linguistique au sens plus strict (traditionnel), on peut la trouver dans plusieurs parties de langue : pronom personnel, déterminant possessif ${ }^{1}$, pronom possessif, adjectif possessif, verbe et même dans l'article selon

1 On trouve aussi d'autres appellations pour les déterminants possessifs tels que: article personnel chez Desseintes (1964) ou déterminant relationnel chez Heinz (2003). 
certains linguistes (par ex. Guéron, 1983). Nous sommes particulièrement intéressés ici de voir comment, à travers les différentes périodes de la langue française, la catégorie de la personne, contenue dans son système, peut influer sur la conception de la personne humaine.

\section{SPHÈRE PERSONNELLE / INALIÉNABILITÉ}

Dans son article de 1926, Bally introduit le terme de sphère personnelle et le définit de la manière suivante : «La sphère personnelle comprend, ou peut comprendre, les choses et les êtres associés à une personne d'une façon habituelle, intime, organique (p. ex. le corps et ses parties, les vêtements, la famille, etc.). Tout élément constitutif de la sphère est considéré, non comme une simple propriété, mais comme une partie intégrante de la personne». (Ibid. 68) Et quelques lignes plus loin il ajoute : « La notion de sphère personnelle est purement subjective; rien n'empêche l'imagination collective d'attribuer au moi des choses ayant leur existence propre, ou inversement, de détacher ce qui ne peut réellement en être séparé (...). L'extension de la sphère personnelle est déterminée par les idées traditionnelles de chaque groupe linguistique; ces limites peuvent varier de langue à langue, varier aussi dans une même langue au cours de son évolution. » (Ibid. 68-69). ${ }^{2}$

Cette définition si vague qu'elle soit renvoie quand même très nettement à la problématique de la conception de la personne dans une société et dans une langue. En d'autres termes elle renvoie indirectement à l'interdépendance des faits inter et extralinguistiques. La conception de la personne dans une société se reflète dans sa langue et la langue par ses mécanismes influe sur la mentalité de cette société.

Aujourd'hui on ne parle plus tellement de la sphère personnelle mais plutôt de l'inaliénabilité (traditionnellement appelée possession inaliénable).

L'inaliénabilité est étroitement liée à la conception de la personne dans une société et dans la langue elle se caractérise par l'emploi d'une morphosyntaxe particulière, qui est différente de la morphosyntaxe de la possession. L'inaliénabilité est le rapport établi (déterminé auparavant) entre la personne et certaines notions considérées comme faisant partie de la personne. L'aliénabilité ou la possession est la relation qu'on établit entre la personne et n'importe quel autre concept qui ne fait pas partie de la personne. L'inaliénabilité, tout comme la sphère personnelle, porte sur les parties du corps, vêtements, famille, outils mais aussi sur toute autre notion par laquelle on définit une personne dans une société donnée. Dans certaines langues mélanésiennes, comme le dit Lévy-Bruhl, la notion de la personne embrasse aussi l'ombre, les restes d'aliments, les meubles, les armes, la maison... En grec et en latin la sphère de la personne s'étendait aux armes et à l'équipement (casque, javelot, lance, flèches, bouclier, selle), aux bateaux, aux chevaux, au peuple, même au roi... ${ }^{3}$

2 Comparer aussi l'article de Lamiroy et Delbecque, "The possessive dative in Romance and Germanic languages » (1998).

3 Cf. Frei, (1939), « Sylvie est jolie des yeux », p. 191 
La morphosyntaxe particulière de l'inaliénabilité veut dire qu'il y a des langues qui ont développé des morphèmes particuliers qui signifient «l'inaliénable » ${ }^{4}$, ce qui est un cas assez rare parmi les langues, et qu'il y en a d'autres qui ont développé une syntaxe un peu plus particulière (des constructions applicables seulement aux notions considérées comme inaliénables). Dans les langues indo-européennes et dans les langues romanes surtout, on parle de constructions telles que le datif de l'inaliénabilité (trad. datif possessif), l'accusatif de l'inaliénabilité (double accusatif) et autres :

1. Je me lave les mains. Vs *Je lave mes mains. Vs Je lave ma voiture.

2. Il m'a pris par la main. Vs *?Il m'a pris par ma main.

Autrement dit, l'inaliénable et l'aliénable se différencient dans l'emploi des constructions implicites et explicites. Le datif de l'inaliénabilité représente la construction implicite typique tandis que le déterminant possessif est le signe de l'aliénable. ${ }^{5}$

\section{LE LATIN ET LA SPHÈRE PERSONNELLE / L'INALIÉNABLE}

Si l'on veut parler de la conception et de l'expression de la personne dans la langue française, il faut faire un pas en arrière et voir comment étaient les choses dans sa langue mère, le latin.

Le latin, à la différence du français, n'employait pas tellement le possessif. Au moins c'est ce qu'on peut conclure des exemples suivants :

\begin{tabular}{|c|c|c|}
\hline Français & \multirow{10}{*}{ Vs } & Latin \\
\hline 3. Pilate se lava les mains. & & 3'. Pilatus manos lavavit. \\
\hline 4. Il leva la main en signe de protestation. & & 4. In signum interpellationis manum sustulit. \\
\hline 5. Il salua sa mère et son père et se mit en route. & & 5. Matre et patre salutato iter ingressus est. \\
\hline $\begin{array}{l}\text { 6. Auparavant il parla avec son frère de son } \\
\text { départ en France. }\end{array}$ & & $\begin{array}{l}\text { 6. Ante hanc rem de profectione ad Galliam } \\
\text { cum fratre locutus est. }\end{array}$ \\
\hline $\begin{array}{l}\text { 7. Il mit son/un pull/T-shirt/tunique et sortit } \\
\text { de la/sa chambre. }\end{array}$ & & 7'. Tunicula vestita camera egressus est. \\
\hline 8. Ille saisit par le cou et le jeta au sol. & & $\begin{array}{l}\text { 8. Faucibus comprehensis ad pavimentum } \\
\text { eum iacuit. }\end{array}$ \\
\hline 9. Il dégaina son épée et lui trancha la gorge. & & 9'. Gladio e vagína educto iugulum ei praecisit. \\
\hline $\begin{array}{l}\text { 10. Il jeta son bouclier à terre et aida son ami à } \\
\text { se relever. }\end{array}$ & & $\begin{array}{l}\text { 10'. Scuto ad pavimentum iacto amico exsur- } \\
\text { gere succurrit. }\end{array}$ \\
\hline 11. Il grimpa sur son cheval et partit. & & 11'. In aequm ascenso iter ingressus est. \\
\hline
\end{tabular}

$4 \quad$ Les langues telles que le kati (Matasović, 2002) ou le tunica (Benveniste, 1966).

5 Il y a aussi des constructions qui exprimaient l'inaliénabilité mais qui ont disparu au fil de temps: li fils li roi, l'escu Lancelot. Herslund (1977) parle de cette construction implicite et l'appelle construction N1N2 
Lehmann (cf. 2005 : 38-39), en parlant de l'évolution du pronom possessif (déterminant possessif, T. F.), trouve trois façons différentes pour exprimer la possession en latin (au moins en ce qui concerne les notions appartenant à la sphère personnelle) :

A. Le possesseur reste sans expression :

$12 \mathrm{a}$. optimo iure infringatur aula cineris in caput

« à juste titre il faudrait casser un pot à cendres dans ta tête » (Pl. Am. 1034d)

$12 \mathrm{~b}$. Leonida, curre obsecro, patrem juc orato ut ueniat

« Leonidas, cours s'il te plaît, supplie mon père de venir » (Pl. As. 740)

B. Le possesseur est exprimé au dativus sympatheticus ou possessivus :

13 a. capiam coronam mi in caput

« je me mettrai une couronne (dans la tête)» (Pl. Am. 999)

$13 \mathrm{~b}$. patrem atque matrem uiuerent uellem tibi

« je souhaiterais que ton père et ta mère étaient encore en vie » (Pl. Poen. 1066)

C. Le possesseur est exprimé par le pronom possessif. Celui-ci est porteur d'emphase et met le possesseur en évidence par rapport à d'autres possesseurs potentiels :

14 a. em, méum caput contemples, si quidem ex er consultas tua

« tiens, regarde ma tête, si tu veux des conseils pour ton bien » (Pl. As. 538)

$14 \mathrm{~b}$. immo suom patrem illic perdidit

« bien au contraire, c'est lui qui a ruiné son propre père » (Pl. Most. 979)

Dans les exemples de Lehmann on peut voir que le type A (le possesseur reste sans expression) ne s'est pas conservé en français et que dans de telles situations le français emploie obligatoirement le déterminant possessif. Lehmann explique le non emploi du possessif dans ces cas par le contexte qui, au moins en latin, renvoie clairement à « ta tête » (ex. 12 a.) et à « mon père » (ex. 12 b.). Dans nos exemples, mentionnés plus haut, il ne s'agit pas du contexte mais nombreux linguistes ici parlent plutôt des noms relationnels. D'après eux, on pourrait classer dans le groupe des noms relationnels les mots suivants de nos exemples: mains, mère, père, frère, épée, bouclier, cheval mais aussi ami, pull/T-shirt/tunique et chambre. C'est du moins ce qu'on peut conclure sur base des exemples français où l'on a le déterminant possessif. Ou en d'autres termes, on peut dire que la notion de la personne en latin comprenait les parties du corps, la famille, les vêtements, les armes, l'armure, la maison et les personnes telles que : amis, voisins...

Dans les exemples où en latin on emploie le dativus possessivus, en français on a respectivement l'emploi du datif de l'inaliénabilité avec la partie du corps (ex. 13 a.) et le déterminant possessif avec les termes de parenté (ex. 13 b.)

Dans les exemples 14 a. et b., les deux langues emploient le possessif. ${ }^{6}$

6 Certes, il y a d'autres façons d'exprimer la relation entre la personne et les notions qui s'y rattachent normalement comme: Mihi nome est... (« Mon nom est.. ») ou Mihi sunt capilli nigri. (« J'ai les cheveux noirs »). De même, il faudrait mieux analyser la tournure avec le datif de l'inaliénabilité en français qui ne s'employait pas en latin : Il se lave les mains « Manus lavat ». Dans l'exemple 13 b. le dativus possessivus a été employé à cause de la préposition 
Lehmann (2005:39) ajoute « Tandis que toutes les occurrences du pronom possessif chez les dénominations de parties du corps sont emphatiques, chez les termes de parenté on peut trouver plus d'exemples » comme c'est le cas en 14. b.

15. ubi amans adulescens scortum liberet clam suom patrem « où le jeune amant rachète sa maîtresse dans le dos de son père » (Pl. Cap. 1032)

Selon Lehmann (cf. 2005 :43-45), le possessif ne s'emploie pas avec les noms relationnels sauf si l'on veut mettre en relief le rapport entre le possesseur et sa partie du corps ou un des membres de sa famille. ${ }^{7}$ En d'autres termes, le possessif n'est employé que dans le cas de l'emphase ou si le contexte ne suffit pas pour l'identification du possesseur. Il conclut que cette caractéristique ${ }^{8}$ du latin (et d'autres langues indo-européennes) est un principe syntaxique hérité du proto-indo-européen.

\section{L'ANCIEN FRANÇAIS ET LA SPHÈRE PERSONNELLE / L'INALIÉNABLE}

"When we come to the period of Old French, we find that the use of the Poss. ${ }^{9}$ (Possessive, T. F.) has increased a hundred-fold. ${ }^{10}$ », dit A. G. Hatcher dans son article « Il tend les mains vs. il tend ses mains » et fournit les exemples suivants : baisser, torner sun chef; torner sun vis, sa teste; ancliner sa chiere ; tendre ses braz... (1944:477-479):

16. Dunc met sa main en sa chalce vermeille,

Si traist defors un enseigne de paille

Ch. de Wilalme 316-7 (cité d'après Hatcher, 1944 :479)

17. Mes sire Yvains, et par le frein

Menoit le cheval an sa main

Por ce que il li voloit randre

Yv. 2269-70 (cité d'après Hatcher, 1944 :479)

18. Li empereres tent (...) ses mains vers Deu,

Baisset sun chef, si cumencet a penser. ${ }^{11}$

Ch. de Roland 137-8

\footnotetext{
in, et dans l'exemple Mihi nome est... le nom qui représente une notion inaliénable se trouve en position du sujet. De même pour l'exemple $13 \mathrm{~b}$.

7 « le pronom possessif est généralement absent des substantifs relationnels et il est employé, avec eux, pour l'emphase. » (Lehmann, 2005:43)

8 L'absence étendue du pronom possessif des substantifs relationnels.

9 Avec les noms appartenant au groupe de parties du corps. (T. F.)

10 Trad. (T. F.) «Quand on arrive à la période de l'Ancien français, on constate que l'emploi du possessif augmente énormément » (par rapport au latin (T. F.))

11 Traduction en français moderne (G. Moignet, La chanson de Roland, Univers des lettres/Bordas): «L'empereur tend les mains vers Dieu, il baisse la tête, il commence à méditer. »
} 
19. Li empereres en tint sun chef enclin ${ }^{12}$

Ch. de Roland 139

Sa constatation n'est que partiellement acceptée par les historiens du français. Dans les grammaires de l'ancien français on ne parle pas souvent de l'alternance entre l'article et le possessif employés avec les parties du corps ou les termes de parenté. Ce n'est que dans le Manuel du français du moyen âge (Syntaxe de l'ancien français) de Ph. Ménard qu'on peut trouver une confirmation partielle pour la constatation de Hatcher. ${ }^{13}$ Mais pour Ménard, l'emploi du possessif avec les parties du corps n'est qu'une des particularités de l'ancien français : «Pour les parties du corps humain où le rapport de possession est suffisamment clair, alors que le FM se contente de l'article défini, l'AF emploie parfois l'adjectif possessif.

Tendi sa main. (Chanson de Guillaume, 1166)

«Il tend la main.»»

Il faut noter que là où Ménard parle plutôt d'une des particularités de l'ancien français, Hatcher voit une norme dans l'usage du possessif,

«In Old French the procedure of alternation between the two constructions ${ }^{14}$ was, from the point of view of distribution, exactly the reverse of that which prevails today: in the older language the norm was the Possessive. » (Hatcher, 1944a:477). ${ }^{15}$

En outre, Hatcher croit à une alternance entre l'emploi et le non emploi du possessif avec les parties du corps, qui change régulièrement d'une époque de la langue à l'autre ${ }^{16}$ :

«If we go back still farther to Latin, we find again a different situation: In the Classical language and even with Plautus the Poss. was almost unknown. And, if we could go back to pre-historic Latin, it is quite likely that we would find that the Poss. was the original construction » (ibid. 477). ${ }^{17}$

Revenons pour l'instant encore une fois sur la thèse de Lehmann (2005) dans son article sur l'évolution du pronom possessif. Selon lui, le passage du latin au français (et en

12 Traduction en français moderne (G. Moignet, La chanson de Roland, Univers des lettres/Bordas) : «L'empereur tient la tête inclinée. »

13 Comparer aussi Brunot (1953:152-155).

14 Avec le possessif ou avec l'article défini (T. F.).

15 Trad. (T. F.) « En Ancien français la procédure de l'alternation entre les deux constructions, du point de vue de la distribution, était exactement l'inverse de la procédure actuelle : dans la période de l'Ancien français la norme était le possessif. »

16 Comparer quand même ce que Lehmann (2005) dit à propos du possessif en proto-indo-européen.

17 Trad. (T. F.) « Si nous remontons encore plus loin, jusqu'au latin, la situation est encore différente: dans la langue classique, même chez Plaute, le possessif était presque inconnu. Si nous pouvions remonter au latin préhistorique, nous constaterions très vraisemblablement que le possessif était la construction d'origine. » 
général aux langues romanes), c'est-à-dire de l'époque « sans » à l'époque « avec » possessif, se caractérise par la simple grammaticalisation de l'emploi du pronom possessif. L'emphase du pronom possessif latin se perd, il devient plutôt neutre et on l'emploie de plus en plus dans les relations possessives prototypiques. ${ }^{18} \mathrm{Si}$ l'on veut insister sur l'emphase, il faut employer l'adjectif propre (lat. proprius). La neutralisation du possessif, selon Lehmann, a pour conséquence la diminution de l'emploi du datif possessif et l'emploi régulier du pronom possessif dans les relations mentionnées plus haut, ce qui apparaît plus clairement dans les constructions avec les termes de parenté. Dans une certaine mesure, ici coïncident les thèses de Hatcher et de Lehmann, du moins en ce qui concerne la norme et l'emploi du possessif avec les termes de parenté (cf. Lehmann : 45-46).

Ajoutons quand même que Lehmann ne mentionne presque pas l'emploi du datif de l'inaliénabilité avec les parties du corps. Sa seule remarque se trouve dans la note en bas de page : «Pour les parties du corps, par contre, on dit en français (...) encore aujourd' hui comme en latin : baisser les yeux, se casser la jambe. » (Ibid. 46)

Notons encore une fois qu'il faudrait mieux analyser la construction avec le datif de l'inaliénabilité en ancien français et surtout qu'il faudrait voir quand elle apparaît pour la première fois dans les textes du Moyen Âge et avec quels concepts. Et encore une remarque en passant, en latin on dit pedem frangere (sans le pronom réfléchi).

Il faut évoquer ici encore une théorie sur l'emploi de l'article et du possessif en français, celle d'André Rocchetti. En comparant l'évolution de l'article et son emploi avec les concepts de la sphère personnelle en français et en italien, Rocchetti indique, à juste titre, que la personne se trouvant dans le verbe et dans le nom, y joue le rôle principal. La présence de la personne dans le verbe italien et la perte de la personne dans le verbe français (dans les trois premiers rangs du singulier) déterminent la différence principale dans l'emploi de l'article ou du possessif avec des différents concepts.

D’une part, le nom français est devenu « Notion pure, détachée de toute fonction, dégagée de tout lien avec la personne et indépendante de toute quantité » (1987:124) tandis que l'italien a partiellement conservé les catégories qui se trouvaient dans le nom latin. C'est ce qui permet de dire en italien : Gli è morto il padre, une construction dans laquelle en français on doit obligatoirement employer le possessif: Son père est mort. (*Il lui est mort le père. $)^{19}$

D'autre part, la personne se trouvant dans la flexion verbale « suggère tout un ensemble de choses qui lui sont normalement attribuées: aliments, habits, logement, parents proches, outils, instruments, moyens de locomotions, etc. Ces choses ou ces êtres sont en quelque sorte prédéterminés par leur appartenance à la sphère de la personne. »" (Rocchetti, 1987:125)

18 D'après les exemples de Lehmann il est évident que la relation possessive prototypique comprend les parties du corps et les termes de parenté.

19 Pourtant il existe une expression semblable mais archaïque: Il lui est né le fils/la fille. 
L'italien en tant que langue qui a conservé la personne dans la conjugaison n'a pas besoin d'employer le possessif dans nos exemples $(5,6,7,9,10,11)$ :

\begin{tabular}{|c|c|c|}
\hline Français & \multirow{7}{*}{ Vs. } & Italien \\
\hline 5. Il salua sa mère et son père et se mit en route. & & 5". Salutò la madre ed il padre e partì. \\
\hline $\begin{array}{l}\text { 6. Auparavant il parla avec son frère de son } \\
\text { départ en France. }\end{array}$ & & $\begin{array}{l}\text { 6". Prima di ciò, parlò con il fratello della } \\
\text { partenza per la Francia. }\end{array}$ \\
\hline $\begin{array}{l}\text { 7. Il mit son/un pull/T-shirt/tunique et sortit } \\
\text { de la/sa chambre. }\end{array}$ & & 7". Indossò la maglietta e uscì dalla stanza. \\
\hline 9. Il dégaina son épée et lui trancha la gorge. & & 9". Estrasse la spada e gli tagliò la testa. \\
\hline $\begin{array}{l}\text { 10. Il jeta son bouclier à terre et aida son ami à } \\
\text { se relever. }\end{array}$ & & $\begin{array}{l}\text { 10". Lasciò cadere lo scudo [a terra] ed aiutò } \\
\text { l'amico ad alzarsi. }\end{array}$ \\
\hline 11. Il grimpa sur son cheval et partit. & & 11". Montò a cavallo e andò via. \\
\hline
\end{tabular}

\section{LA PERTE DE LA FLEXION VERBALE EN ANCIEN FRANÇAIS ET SES CONSÉQUENCES POUR LA SPHĖRE PERSONNELLE}

On peut décrire la phase de l'ancien français comme une période mixte au moins en ce qui concerne l'alternance dans l'emploi du possessif et de l'article défini avec les concepts appartenant à la sphère personnelle ou inaliénables. On peut trouver les mêmes concepts dans des constructions identiques où les concepts inaliénables sont tantôt employés avec le possessif tantôt sans lui. De même il y a des cas où des concepts considérés aujourd'hui comme aliénables apparaissent soit avec soit sans possessif.

Comparons les exemples suivants : ${ }^{20}$

\begin{tabular}{|c|c|c|}
\hline \multicolumn{1}{|c|}{ Article } & \multicolumn{1}{|c|}{ Possessif } \\
\cline { 1 - 1 } $\begin{array}{l}\text { 20. Quant le vit Guenes, mist la main a } \\
\text { l'espee } \\
\text { Ch. de Roland 443 }\end{array}$ & $\begin{array}{l}\text { 21. Li arcevesque ne poet muer n'en plurt, } \\
\text { Lievet sa main, fait sa b[en]eïçun } \\
\text { Ch. de Roland 2193-4 }\end{array}$ \\
\cline { 1 - 1 } $\begin{array}{l}\text { 22. Pur ses pecchez Deu (recleimet) en } \\
\text { puroffrid lo guant. } \\
\text { Ch. de Roland 2365 }\end{array}$ & $\begin{array}{l}\text { 23. Si l'en enveiet sun bastun e sun guant. } \\
\text { Ch. de Roland 2727 }\end{array}$ \\
\cline { 1 - 1 } $\begin{array}{l}\text { 24. Quant l'oït Guenes, l'espee en ad branlie; } \\
\text { Ch. de Roland 499 }\end{array}$ & $\begin{array}{l}\text { 25. Desuz lui met s'espee e l'olifan } \\
\text { Ch. de Roland 2359 }\end{array}$ \\
\hline
\end{tabular}

La question centrale est de voir pourquoi et comment on est arrivé à l'emploi du possessif avec les inaliénables, c'est-à-dire avec les noms qui en latin ont été employés sans possessif, et qui sont considérés comme appartenant à la sphère personnelle. Ce moment

20 Traduction en français moderne (G. Moignet, La chanson de Roland, Univers des lettres/Bordas): «pour ses péchés il tend vers Dieu son gant. » 
nous paraît très important non seulement pour l'explication de l'emploi du possessif avec différents concepts (peu importe s'ils appartiennent ou pas à la sphère personnelle) mais aussi pour la catégorie de la personne en général ainsi que pour le système du pronom personnel en particulier.

On sait que la période de l'ancien français est une période de bouleversement dans le système du français. D’une part, on a des changements sur le plan nominal : la déclinaison est en train de disparaître, le nom devient une notion pure (Rocchetti), on assiste à l'apparition de l'article, on introduit le possessif avec les noms inaliénables... D'autre part, sur le plan verbal, on rencontre aussi des changements significatifs. La conjugaison se perd (partiellement), ce qui a pour conséquence directe l'introduction obligatoire et systématique du pronom personnel détaché et antéposé au verbe.

Cela peut sembler étrange, mais pour les concepts inaliénables, l'étude de la personne du verbe est plus importante que l'étude de la personne dans le nom. En effet le nom garde la catégorie de la (troisième) personne tandis que le verbe la perd.

Revol dit que « L'étude des désinences de l'indicatif présent est essentielle pour l'ensemble du système morphologique du verbe » (2005:125). Nous sommes tout à fait d'accord avec son avis sauf que nous l'élargissons aussi à l'étude de la personne.

La différence entre les trois premières personnes du singulier dans tous les groupes verbaux disparaît et le système verbal commence à perdre en clarté (au moins à l'oral). De l'importance de ce fait témoignent les phrases suivantes : « Les désinences s'étaient bien conservées jusqu'à la fin du XIII ${ }^{\mathrm{e}}$ siècle parce que leur fonction informative était importante dans une langue, où la présence du pronom personnel n'était pas obligatoire. Aujourd'hui, de nombreuses formes d'indicatif présent sont confondues à l'oral... mais la présence du pronom, de plus en plus systématique à partir du XIII ${ }^{\mathrm{e}}$ siècle, vient compenser cette faiblesse du système. » (Revol, $2005:$ 127-128)

Le $-s$ de la première personne et le $-t$ de la troisième personne se trouvent sporadiquement dans les textes du XII ${ }^{e}$ et du XIII ${ }^{e}$ siècle mais dès ce temps-là elles ne sont plus prononcées et sont en train de disparaître. Le $-s$ final de la deuxième personne ne s'est conservé que dans la graphie.

Tout cela fait que le système devient de moins en moins compréhensible et la nouvelle situation force le système à réagir. Le remède est vite trouvé. Et il est bien logique. A la disparition de la personne de la conjugaison verbale du présent de l'indicatif (les trois premières personnes) la langue « répond » par l'introduction obligatoire du pronom personnel qui au fur et à mesure devient la partie obligatoire du syntagme verbal.

Mais le processus ne s'arrête pas là. Il serait faux de conclure simplement que l'introduction du pronom personnel et son emploi obligatoire devant le verbe signifient la fin des changements dans le système verbal qui concernent la personne et le pronom personnel.

En effet, il y a une très grande différence entre la personne conservée dans la désinence du verbe et celle qui en est détachée. 
Le verbe exprime une action ou un procès. C'est ce qui se trouve dans son radical. Dans sa désinence se trouve l'indication du temps et de la personne. Le français garde cette indication pour tous les temps sauf pour l'indicatif du présent. En effet, cette action se déroule au moment de la parole, et pour cela elle est immédiatement reconnue comme actuelle, c'est-à-dire comme faisant partie du (temps) présent. Pourtant la perte de la personne doit être « compensée ».

La connexion entre le verbe et la personne dans une forme synthétique fait de cette personne un actant agissant, participant immédiatement à l'action exprimée par le verbe. Ce fait lui donne la possibilité d'agir et d'élargir son influence sur son entourage. C'est pour cette raison que le latin n'avait besoin ni du pronom personnel (pour indiquer qui exerce l'action), ni non plus du déterminant possessif (pour indiquer à qui appartient l'objet se trouvant dans la phrase). Rappelons que plus ou moins la même chose vaut pour l'italien (comp. plus haut la citation de Rocchetti sur les aliments, habits, logement, parents proches...).

Reprenons encore une fois nos exemples $n^{\circ} 5$ et 10 :

\begin{tabular}{|c|c|c|c|}
\hline Français & \multirow{3}{*}{ Vs } & Latin & Italien \\
\hline $\begin{array}{l}\text { 5. II salua sa mère et son } \\
\text { père et se mit en route. }\end{array}$ & & $\begin{array}{l}\text { 5. Matre et patre salutato } \\
\text { iter ingressus est. }\end{array}$ & $\begin{array}{l}\text { 5". Salutò la madre ed il pa- } \\
\text { dre e partì. }\end{array}$ \\
\hline $\begin{array}{l}\text { 10. Il jeta son bouclier à terre } \\
\text { et aida son ami à se relever. }\end{array}$ & & $\begin{array}{l}\text { 10' Scuto ad pavimentum ia- } \\
\text { cto amico exsurgere suc- } \\
\text { currit. }\end{array}$ & $\begin{array}{l}\text { 10" Lasciò cadere lo scudo [a } \\
\text { terra] ed aiutò l'amico ad } \\
\text { alzarsi. }\end{array}$ \\
\hline
\end{tabular}

La personne dans le verbe (appelons-la la personne intraverbale) indique immédiatement qui est le porteur de l'action exprimée par le verbe. A la différence de cette personne intraverbale, la personne détachée du verbe (la personne extraverbale) doit indiquer explicitement qui est porteur de l'action, et en plus, elle doit lier cette personne avec le verbe. ${ }^{21}$ Elle fait une (ou même deux) action en plus par rapport à la personne intraverbale. Cette caractéristique affaiblit son impact et son influence sur son entourage, et elle devient une sorte de personne non-expressive. Cette personne non-expressive est liée à la fonction de représentation du porteur de l'action du verbe. Elle n'a pas ni l'autonomie ni le pouvoir qu'avait la personne intraverbale. Sa capacité d'influer sur son entourage se perd dans le processus de rattachement au verbe, et elle devient tellement faible qu'il faut introduire des éléments explicites (le déterminant possessif) pour indiquer à qui appartient l'objet de la phrase, même s'il n'y a qu'une seule personne dans la phrase. Il faut renforcer cette personne affaiblie suite à la perte de la flexion verbale, par l'introduction du possessif qui conserve la catégorie de la personne :

21 Comp. l'exemple Alfred parle. vs *Alfred parlons. ou pour mieux visualiser la problématique de la personne, changeons le nom par le pronom : Il parle. vs *Ils parlons. 
26. Par amistiet l'en baisat en la buche,

Si l'en dunat s'espee e s'escarbuncle.

Ch. de Roland 1530-1

27. Sur l'erbe verte s'i est culcet adenz,

Desuz lui met s'espee e l'olifan;

Ch. de Roland 2358-9

Pourquoi, alors, n'y a-t-il pas de possessif avec les parties du corps ?

Avec les parties du corps la situation est juste un peu différente. Au début de ces changements sur le plan verbal on a commencé à employer les possessifs avec les parties du corps (d'où le « hundred-fold» de Hatcher). Brunot dit que : « En a. f. on trouve le plus souvent le possessif à sa place normale... Toutefois l'article était déjà commun avec les noms des parties du corps... La différence entre article et possessif est si faible qu'on les voit alterner... Desuz le frunt li buillit la cervele... Desur sun piz... » (1953 : 153-154 $)^{22}$.

Dans un certain sens, le système devient instable car il autorise deux possibilités : l'article ou/et le déterminant possessif avec les parties du corps. D'un côté, le verbe perd la personne dans la conjugaison et on commence à introduire le possessif (pour renforcer la personne affaiblie) comme le signe normal de la possession, mais de l'autre côté, les parties du corps sont la personne. D'où la confusion et l'alternance entre l'article et le possessif avec les parties du corps en ancien français. Il se posait la question s'il fallait employer un élément explicite comme le possessif pour marquer le rapport entre la personne et ses parties. Rappelons ici les paroles de Lévy-Bruhl qui dit : «Quand je parle de ma tête, je ne veux pas dire qu'elle est à moi, mais qu'elle est moi. » (1916 : 98). Les parties du corps ont en eux-mêmes la prédétermination naturelle d'être inaliénables. C'est notre conception de la personne, née avec ses parties du corps. Ce fait a été décisif et les parties du corps ${ }^{23} \operatorname{sont}^{2}$ restées le seul concept avec lequel on n'employait normalement pas le possessif.

Donc, la personne intraverbale exerce plus facilement une influence sur son entourage (sur sa sphère personnelle) tandis que la personne extraverbale est moins forte et perd sa force en se rattachant au verbe. Son premier rôle est d'indiquer le lien avec verbe.

C'est pour cette raison que la personne extraverbale ne peut influer que sur les parties du corps. Elle est affaiblie de telle façon, que pour tous les autres concepts, il faut renforcer l'influence de la personne en introduisant le déterminant possessif (élément explicite de la possession) pour qu'il rétablisse la relation entre le possesseur et le possédé.

Tout ce bouleversement sur le plan verbal, personnel et pronominal perdure jusqu'à plus ou moins la fin du XIII ${ }^{\mathrm{e}}$ siècle quand on arrive au moyen français, une période de relative stabilisation.

22 Voir aussi les exemples 20 et 21.

23 Et d'autres concepts considérés comme faisant partie intégrante de la personne: vie, idées, voix, température... 


\section{LE MOYEN FRANÇAIS, LE FRANÇAIS CLASSIQUE ET LA SPHÈRE PERSONNELLE / L'INALIÉNABLE : VERS UNE STABILISATION ?}

On sait que la langue change perpétuellement et que d'un point de vue diachronique on peut toujours parler d'une phase de la langue (qui change). Pendant la période du moyen français, on favorise la fixation du pronom sujet, on remplace le CR li par lui, on substitue aux possessifs féminins non expressifs $m^{\prime}, t^{\prime}, s^{\prime}$ devant une voyelle les formes masculines mon, ton, son, on « rationalise » le marquage désinentiel : on a quatre formes pour les verbes du premier groupe...

Bien que la situation en moyen français ne soit pas encore complètement stabilisée, on voit, quand même, une tendance vers la normalisation de l'emploi de l'article et du possessif dans les syntagmes avec les parties du corps. Dans de telles situations on commence à préférer l'article.

28. L'infante.

Mets la main sur mon cœur, (Corneille, Le Cid: I,2)

29. Don Rodrigue.

Cette ardeur que dans les yeux je porte, sais-tu que c'est son sang ? Le sais-tu? (Corneille, Le Cid : II,2)

30. Félix

Donne la main, Pauline. (Corneille, Polyeucte, VI)

C'est l'époque où les premières règles commencent à apparaître. Il faut passer la Manche pour lire dans la grammaire de John Palsgrave (L'esclaircissement de la Langue Françoyse, 1530) une règle sur l'emploi de l'article avec les parties du corps : «For where as we (les anglophones, T. F.) say: he hurteth my hande; I cut my finger; she dyd put out her eie; they (les francophones, T. F.) say: he me hurteth the hande; I me cut the finger; she her dyd put out the eie.» (cité d'après Spanoghe, $1995: 73$ ). ${ }^{24}$

Il est clair que l'auteur explique la différence dans l'emploi de l'article devant une partie du corps en français et l'emploi obligatoire du possessif dans les mêmes situations en anglais.

En France, il faut attendre l'œuvre posthume de Vaugelas, Nouvelles remarques de M. de Vaugelas sur la langue françoise (1690), pour indiquer que le possessif dans le syntagme avec une partie du corps « est mal parler»: «Un loup enleva un enfant sans entamer sa peau. Je dis que c'est mal parler, \& qu'il faut dire Un loup enleva un enfant sans luy entamer la peau. » (cité d'après Heinz, 2003 : 74). Un peu plus loin quand il s'agit des habits on lit : «Un loup enleva un enfant sans luy entamer la peau \& sans déchirer

24 La règle de Palsgrave: „Regula prima: Whan we expresse dymynisshyng or hurting or generally any acte to be done to any parte of a man or beestees body, in all such sentences, they resolve the pronoune deryvatyve in to his primytyve, usynge the article le int he place where the pronounederyvatyve was used in our tonge... The hangman dyd fyrst bynde his eyes and after dyd cutte of his heed: le bourreau primier luy benda les yeulx et puis luy couppa la teste" (cité d'après Spanoghe, 1995 : 73). 
ses habits, \& non-pas sans luy déchirer les habits. \) L'explication qui suit (qu'habits n'est point équivoque et qu'il ne convient qu'à l'enfant) nous parait quand même un peu superficielle. Même si on passe très souvent sous silence que l'emploi du possessif avec les concepts autres que les parties du corps devient la règle, il faut mentionner ici que la tournure se déchirer les habits ne se dit pas même si le contexte n'est pas moins clair comme le disait Vaugelas. En effet, la tendance était de ne pas employer le datif de l'inaliénabilité (qui est une sorte d'adaptation du dativus possessivus latin en ancien français) avec les vêtements. Le datif de l'inaliénabilité paraît comme une certaine réinvention de la réflexivité avec les parties du corps. En outre, la forme réfléchie avec les vêtements a été très rare en ancien français. On trouve le plus souvent les exemples de type :

31. Fiert l'amiraill de l'espee de France,

L'elme li freint o li gemme reflambent,

[T]renchet la teste pur la cervele espandre

Ch. de Roland 3615-7

Après la période du moyen français, et du français classique surtout, on aura d'une part une quasi stabilisation de l'emploi de l'article avec les parties du corps, du moins en ce qui concerne la norme. Mais d'autre part, il semble que les auteurs classiques ont eu un goût particulier pour le possessif. Dans deux éditions du Bon Usage $\left(10^{\mathrm{e}}\right.$ et $\left.13^{\mathrm{e}}\right)$ on trouve des exemples : Il frotte SES mains (La Bruyère); Il étend SES bras (Diderot); J'ay beau frotter MON front, j'ay beau mordre MES doigts (Boileau). Tandis que dans la $13^{\mathrm{e}}$ édition on ne trouve aucune explication, dans la $10^{\mathrm{e}}$ édition on explique ce phénomène par la volonté de souligner la personnalisation de celui dont il s'agit (LBU ${ }^{10}:$ $\$ 427$ a) Remarque).

Selon Brunot (1953 : 154) «Autour de 1600, l'usage normal était déjà de considérer l'article comme suffisant quand il s'agissait des diverses parties du corps ». Pourtant le goût pour le possessif chez les classiques est, selon lui, la conséquence de la non publication du dudit livre de Vaugelas ${ }^{25}$ et l'ignorance des règles rigides proscrites par les grammairiens du XVIIe siècle (ou la résistance à celles-ci).

Pourtant, on stabilise par ailleurs l'emploi du possessif avec tous les autres concepts qui jadis faisaient partie de la sphère personnelle :

32. Jean Lyon tout premier, monté sur un cheval morel; et mit tantôt pied à terre, et prit sa hache en sa main. (Jehan Froissart, Chroniques, Livre Second, chapitre LVI)

33. Parquoy le serviteur desguaina son espée et... (Plutarque, Antonius, p. 415, trad. par Jacques Amyot)

et on trouve rarement des exemples de type

34. Quant l'oït Guenes, l'espee en ad branlie;

Ch. de Roland 499 


\section{LE FRANÇAIS MODERNE ET LA SPHÈRE PERSONNELLE / L'INALIÉNABILITÉ}

Quand on vient à la période du français moderne, on trouve une grande quantité de règles sur l'emploi de l'article ou du possessif avec les parties du corps (et les facultés de l'esprit). De nouveau, on ne trouve pas de règles qui concernent les autres concepts. On sous-entend, paraît-il, qu'il faut les employer avec le possessif.

Ces règles sont trop nombreuses pour qu'on les énumère ici mais mentionnons seulement que Sandfeld (1965) dans sa Syntaxe du français contemporain cite 5 règles principales qui se divisent en plusieurs sous règles. Hatcher (1944) parle de 6 types de phrases où l'on peut employer soit l'article soit le possessif. Brunot (1953 [1926]) y consacre un chapitre, Heinz (2003) plusieurs chapitres, Spanoghe (1995) le livre entier.

Soulignons quand même que les linguistes citent souvent une différence cruciale dans l'emploi de l'article et du possessif avec les parties du corps : l'article est le signe du neutre (normal), le possessif est le signe de l'emphase. Cette différence correspond presque tout à fait à la thèse de Lehmann pour l'emploi du possessif avec les termes de l'inaliénabilité en latin.

Pourtant il est très difficile de donner une règle pertinente puisqu'il semble que dans de nombreuses situations l'emploi dépende du goût de l'auteur/locuteur ou des raisons pragmo-sémantiques. Heinz (2003:103) trouve six façons différentes pour dire une seule chose ${ }^{26}$ :

35.

a) croiser les bras sur sa poitrine

b) croiser ses bras sur sa poitrine

c) croiser les bras sur la poitrine

d) croiser ses bras sur la poitrine

e) se croiser les bras sur la poitrine

f) se croiser les bras sur sa poitrine

Les explications sont souvent de type : « Le geste est décrit comme une attitude... le mouvement n'a que peu d'importance... Les gestes sont présentés comme figés... 'sa poitrine' est alors ressenti comme pléonastique... les deux parties du corps impliquées dans le geste sont estompées...»

A côté de ces raisons Brunot voit encore « les caprices de langue : j’y perdrai la vie, la raison ; j'y perdrai mes cheveux » ainsi qu'un usage libre d'écrivains : " Ainsi Lamartine a écrit : La biche impatiente au vent tendait sa tête» $(1953: 155)$. Hatcher parle d'une affinité particulière pour l'article ou pour le possessif chez certains auteurs : « Indeed this author [Martin du Gard] seems to have a particular affinity for the article, as Flaubert had for the Poss. » (1944:471-472, note 19). ${ }^{27}$

26 Ses exemples sont tirés de la base FRANTEXT.

27 Trad. (T. F.) «En effet, il semble que cet auteur (Martin du Gard) avait le goût de l'article, tout comme Flaubert avait celui du possessif. » 
On peut ajouter ici aussi que les traductions des textes peuvent varier d'un traducteur à l'autre (ou peut-être d'un siècle à l'autre). Ainsi dans la traduction de La Bible (Le Nouveau Testament) de Martin (1744) on trouve que :

36. Pilate... prit de l'eau, et lava ses mains... (Mathieu 27:24)

tandis que dans les traductions de Darby (1859) et de Louis Segond (1910) on trouve que:

37. Pilate... prit de l'eau, se lava les mains... (Mathieu 27:24)

On voit que les traductions plus récentes (Darby $\mathrm{XIX}^{\mathrm{e}}$ siècle, Louis Segond $\mathrm{XX}^{\mathrm{e}}$ siècle) sont plus conformes aux règles prescrites par les grammairiens sur l'emploi du datif de l'inaliénabilité avec les parties du corps, et que dans la traduction de Martin (XVIII siècle) on trouve toujours le possessif.

Toutes ces règles sur l'emploi ou non de l'article, qui se rapportent plus ou moins exclusivement aux parties du corps, nous mènent à conclure que la sphère personnelle en français s'est rétrécie au cours de l'histoire au seul corps humain (avec une extension vers les facultés d'esprit ; se torturer l'esprit (Bally, 1926 :77)). Dans un certain sens elles confirment les paroles de Bally: «Ainsi le français d'aujourd'hui restreint le domaine du moi subjectif au corps et à l'esprit du sujet. » (1926 :77). Frei (1939:191-192) explique ce rétrécissement du moi (de la personne) par le changement de la mentalité : «A cause du rétrécissement de la notion de personne, nos langues, le français du moins, ne peuvent pas dire « agité de casque », « fameux de lance », « brillant de selle »... » comme c'était le cas en grec. « L'application différente de la règle de convertibilité ${ }^{28}$, aujourd'hui et dans le passé, suggère donc un changement de mentalité ». (Ibid. 192) ${ }^{29}$

Ajoutons que tout cela contribue aussi à l'idée de Bally qui rattache la sphère personnelle exclusivement à la vie organique c'est-à-dire aux seuls êtres humains ou aux choses personnifiées.

Mais il n'est pas pourtant ainsi, au moins en français moderne. Ni la sphère personnelle n'est limitée aux parties du corps, ni les êtres vivants (humains ou animaux) ${ }^{30}$ ne bénéficient pas du droit d'avoir l'exclusivité pour la sphère personnelle.

Si l'on admet que le datif de l'inaliénabilité est un bon signe de la sphère personnelle (de l'inaliénable) il faut noter qu'on trouve des exemples de datif de l'inaliénabilité avec d'autres concepts : vêtements, accessoires, famille... Ces exemples sont beaucoup moins nombreux que ceux avec les parties du corps, mais cela ne diminue pas leur importance.

28 C'est-à-dire la possibilité d'employer la construction implicite.

29 Pour le changement de la mentalité Frei cite un exemple de Ronsard:

„Je te salue, ô terre plantureuse,

Heureuse en peuple et en princes heureuse,“

et il ajoute que „il y a quelque chose qui ne semble pas en règle. C'est que, pour un moderne, la terre et le peuple qui l'habite, le sol et les rois qui le possèdent ne sont pas conçus comme identique de substance." (1939: 192)

30 Voir note en bas de page $n^{\circ} 16$. Regula prima... any acte to be done to any parte of a man or beestees body... 
Vêtements :

38. Au passage, les branches des groseilliers sauvages nous agrippaient par la manche. (Le Grand Meaulnes : 227)

39. il s'est mouillé les chaussures. (Herslund, 1983)

Accessoires :

40. Ils nous fouillent dans les sacs.

41. Il met à nu Durendal, sa bonne épée (...)

Lui brise le heaume ${ }^{31}$

Ch. de Roland 1324-6

Famille :

42. Il lui est née la fille (arch.)

Dans tous ces exemples, on sent que la personne est fortement impliquée dans l'action du verbe. C'est toujours la personne qui est en question. Les autres concepts ne sont là que pour aider à décrire l'état où se trouve la personne. Ainsi, ce n'est pas la manche ou le col qui est pris mais plutôt la personne qui les porte. Les sacs qui sont fouillés sont portés par les personnes (qui veulent entrer par exemple à la Sorbonne). Dans le dernier exemple nous avons le verbe naître qui normalement n'exige pas un complément indirect, ce qui fait que la personne au datif (lui) est vue comme une mise en évidence.

De manière similaire, d'autres concepts peuvent avoir leur sphère personnelle, toujours exprimée par le datif de l'inaliénabilité :

43. «Celui-là, disait-elle, les Prussiens lui ont cassé les anses, en soixante-dix, parce qu'ils ne pouvaient pas l'emporter». (Le Grand Meaulnes : 212 ; lui $=$ vase, n. m.)

44. Si l'arbre s'étend trop, vous devez lui tailler les branches. (Borillo 1999:66, note 9)

45. ...parasites qui lui grimpent jusqu'au bout des ailes. (A. Daudet, Lettres de mon moulin : 9 ; lui = moulin, n. m.)

46. Notre voiture eut à souffrir (...) tantôt lui crevait deux pneus. (Express 1840.55 , le 17.10 .86$)^{32}$

On peut voir que la sphère personnelle peut être appliquée aux concepts non vivants et que dans nos exemples ils ne sont guère personnifiés.

31 Traduction en français moderne (G. Moignet, La chanson de Roland, Univers des lettres/Bordas). On trouve aussi la version avec le possessif : Il lui brise son heaume.

32 Cité d'après Herslund, 1983 


\section{CONCLUSION}

Dans notre travail nous avons essayé de montrer comment la notion grammaticale de la personne influe sur la conception de la personne dans une langue. Nous avons également fourni une explication possible de l'emploi du possessif avec les concepts autres que les parties du corps qui jadis faisaient partie de la sphère personnelle.

On est parti du latin qui n'employait presque pas le possessif avec les termes faisant partie de la sphère personnelle : parties du corps, famille, armures, vêtement... pour arriver à l'époque du français moderne qui a rétréci la notion de la personne à ses seules parties du corps. A travers les différentes phases de la langue française on a essayé de montrer comment on est arrivé à ce stade de l'emploi surabondant du possessif et d'une relative stabilisation de l'emploi de l'article avec les parties du corps.

La période la plus importante, selon nous, était celle de l'ancien français et des changements sur le plan nominal et surtout sur le plan verbal. La perte de la conjugaison et en même temps de la personne se trouvant dans la flexion verbale a exercé une grande influence sur le système du verbe mais aussi sur celui de la personne. Quand on est passé de la phase où la personne faisait partie du verbe (la personne intraverbale) à la phase où la personne en était détachée (la personne extraverbale) on a noté deux faits : premièrement, le pronom personnel devient l'élément obligatoire de tout syntagme verbal et deuxièmement, on emploie systématiquement le possessif avec tous les autres concepts sauf les parties du corps. Ces faits sont la conséquence directe de l'affaiblissement de l'influence de la personne qui se trouvait dans la flexion verbale lors de son passage à l'extérieur du verbe où elle ne pouvait plus exercer son influence sur les notions se trouvant dans la phrase (contexte).

Après cette période de bouleversement on est arrivé à la période où l'on a commencé à fixer les premières règles sur l'emploi de l'article avec les parties du corps. Malgré les 500 ans de tradition de fixation des règles, il faut avouer qu'on peut toujours se poser des questions quand on se trouve devant des syntagmes tels que : (se) croiser les/ses bras sur la/sa poitrine. Des explications de l'emploi de l'article ou du possessif tiennent toujours compte de la situation ou du contexte pragmo-sémantique.

D'un côté, l'alternance entre l'article et le possessif avec les parties du corps, et de l'autre côté, l'emploi du possessif avec les termes qui jadis faisaient partie de la sphère personnelle, ont eu une conséquence générale : à travers les siècles la notion du moi (de la personne) s'est rétrécie aux parties du corps et aux facultés de l'esprit (malgré le fait qu'on peut trouver des exemples (beaucoup moins nombreux) avec d'autres concepts). Les uns y voient le changement de la mentalité de l'homme moderne (Frei), les autres parlent de la grammaticalisation de l'emploi du possessif (Lehmann), Hatcher voit l'alternance régulière dans l'emploi du possessif qui change d'une phase de la langue à l'autre (latin pré-historique + Poss $\rightarrow$ latin classique - Poss $\rightarrow$ ancien français + Poss $\rightarrow$ 
français moderne -Poss). Rocchetti parle de l'implication de la personne dans le système nominal et verbal.

Notre position est plus proche de celle de Rocchetti et nous avons essayé de montrer que c'est le changement sur le plan verbal qui a eu pour conséquence la perte (partielle) de la personne, qui a mené « au rétrécissement» du moi moderne.

Mais il semble que l'emploi du possessif s'est tellement répandu qu'aujourd'hui on l'emploie dans presque n'importe quelle situation. Voici quelques exemples :

47. Tu as fait ta (la) vaisselle.

48. Tu vois, tu ne diras jamais ça à ton (un) professeur.

49. Luc a acheté son (un) costume.

50. Ce ticket sera exigé pour reprendre votre réparation.

51. Trouvez facilement votre (un) logement.

52. a) Introduisez votre (la) monnaie. b) Composez votre (le) numéro sur le clavier. c) Prenez votre (la) monnaie, si vous n'avez pas fait l'appoint. d) Dégustez votre (le) produit.

Dans ces cas on pourrait parler d'un certain abus du possessif, mais c'est déjà une autre histoire. ${ }^{33}$

\section{BIBLIOGRAPHIE}

BALly, Charles (1926) L'expression des idées de sphère personnelle et de solidarité dans les langues indo-européennes, in Festschrift Louis Gauchat, Aarau Sauerlander, V. von H.R., p. 68-78.

Banniard, Michel (1997) Du latin aux langues romanes, Paris : Nathan.

Benveniste, Emile (1966) Problèmes de linguistique générale, Paris : Gallimard.

Borillo, Andrée. (1999) Partition et localisation spatiale : les noms de localisation interne. Langages, ${ }^{\circ} 136,53-75$.

Brunot, Ferdinand $\left(1953^{3}\right)$ La pensée et la langue. Paris : Masson et $\mathrm{C}^{\mathrm{ie}}$.

CAstex, Pierre-George/Paul Surer /George Becker (1967) Manuel des études littéraires françaises, Moyen Age. Paris : Hachette.

CAtach, Nina $\left(1998^{8}\right.$ ) L'orthographe. (Que sais-je?). Paris : PUF.

Cerquiglini, Bernard (1993²) La naissance du français. (Que sais-je?) Paris : PUF. DauZAT, Albert (1956) Les étapes de la langue française. (Que sais-je?) Paris : PUF.

33 Je tiens à remercier Samir Bajrić de m'avoir fourni les exemples 47 - 50. Egalement, mes remerciements vont aux personnes suivantes pour leurs traductions : Robert Leljak (latin), Angelo Malangone (italien), Anthony Nichols (anglais), Julien Hintzy et Régis Pavat (français). Je tiens à exprimer mes remerciements les plus profonds à JeanFrançois Brackman pour ses traductions en français et surtout pour le temps qu'il a mis de lire, relire et de rédiger ce texte. 
Foulet, Lucien $\left(1970^{3}\right)$ Petite syntaxe de l'ancien français. Paris : Librairie Honoré Champion. FreI, Henri (1939) Sylvie est jolie des yeux, Mélanges de linguistique offerts à Charles Bally, Genève : Librairie de l'Université, 185-192.

FrletA, Tomislav (2007), Quelques termes concernant l'inaliénabilité, Actes du $4^{\text {ème }}$ Colloque sur les études françaises en Croatie E. Le Calvé-Ivičević (éd.). Zagreb : Artresor naklada, 81-89.

Grevisse, Maurice (1993) Le bon usage. (13 ${ }^{\text {ème }}$ èdition, refondue par André Goosse) Paris : Duculot.

Grevisse, Maurice (1975) Le bon usage. (10 ème édition) Paris : Duculot, Gembloux.

GuÉron, Jacqueline. (1983) L'emploi 'possessif' de l'article défini en français. Langue française 58, 23-35.

Hatcher, Anna Granville. (1944) Il tend les mains vs. Il tend ses mains. Studies in Philology 41, 457-481.

HeINZ, Michaela (2003) Le possessif en français: Aspects sémantiques et pragmatiques. Bruxelles : De Boeck.Duculot.

Herslund, Michael (1983) Le datif de la possession inaliénable en français. (M. Herslund, O. Mørdrup, F. Sørensen, eds). Revue romane 24, u Analyses grammaticales du français. Etudes publiées à l'occasion du $50^{e}$ anniversaire de Carl Vikner. Copenhague : Etudes Romanes de l'Université de Copenhague 24, Copenhague, Akademisk Forlag, 99-115.

Herslund, Michael (1977) L'expression de la "possession inaliénable” en ancien français. L. Carlsson (ed.). Actes du $\sigma^{e}$ Congrès des Romanistes Scandinaves, Studia Romanica Upsaliensia 18, 125-134.

Huchon, Mireille (2002) Histoire de la langue française. Québec : Librairie Générale Française.

LAmiroy, Beatrice/Nicole Delbecque (1998) The possessive dative in Romance and Germanic languages. W. Van Langendonck, W. Van Belle (eds) u The dative, vol 2: Theoretical and contrastive studies. Amsterdam/Philadelphia : John Benjamins Publishing Company, 29-74.

Lancelot, Claude (2000) Port-Royal: Opća i obrazložbena gramatika (Grammaire générale et raisonnée de Port-Royal, trad. en croate par Vojmir Vinja). Zagreb : Institut za hrvatski jezik i jezikoslovlje.

Lehmann, Christian (2005) Sur l'évolution du pronom possessif. S. Kiss, L. Mondin, G. Salvi (éds.), Latin et langues romanes. Etudes de linguistiques offertes à Jozsef Herman à l'occasion de son $80^{\text {ème }}$ anniversaire. Tübingen : M. Niemeyer.

LEVy-BruHL, Lucien. (1916) L'expression de la possession dans les langues mélanesiennes. Mémoires de la société linguistique de Paris 19, 2, 96-104.

Matasović, Ranko (2002) Otuđiva i neotuđiva posvojnost u hrvatskome jeziku. Rasprave Instituta za hrvatski jezik i jezikoslovlje 28, 151-160. 
MÉNARD, Philippe (1973) Manuel du français du moyen âge, 1. Syntaxe de l'ancien français. Bordeaux : Sobodi.

Moignet, Gérard (1973) Grammaire de l'ancien français, morphologie, syntaxe. Paris : Editions Klinsieck.

Moignet, Gérard (1981) Systématique de la langue française. Paris : Klincksieck.

Revol, Thierry (2005) Introduction à l'ancien français. Paris : Armand Colin.

Rocchetтi, André (1987) Théorie de l'article en vue d'une étude contrastive italien/ français. Chroniques italiennes 11/12, 105-151.

SANDFELd, Kristian (1965) Syntaxe du français contemporain. Paris : Droz.

Spanoghe, Anne-Marie (1995) La syntaxe de l'appartenance inaliénable en français, en espagnol et en portugais. Frankfurt am Main : Peter Lang.

TESNIĖRE, Lucien (1988 [1959]) Eléments de syntaxe structurale. Paris : Editions Klincksieck. ZINK, Gaston $\left(2000^{5}\right)$ L'ancien français. (Que sais-je ?). Paris : PUF.

ZINK, Gaston (1990) Le moyen français (Que sais-je?). Paris : PUF.

\section{Textes dépouillés}

Alain-Fournier (1971) Le Grand Meaulnes. Paris : Librairie Fayard.

Chrétien de Troyes (1936) Euvres choisies. Paris : Classiques Larousse.

Corneille, Pierre (1967) Le Cid. Paris : Bordas.

Corneille, Pierre (1965) Polyeucte. Paris : Bordas.

DAUdET, Alphonse (1979) Lettres de mon moulin. Paris : Gallimard.

Froissart, Jehan Chroniques. Livre Seconde, source internet : http://users.skynet.be/antoine.mechelynck/chroniq/froiss/F0.htm\#F2.

Henry, Albert (1953) Chrestomathie de la littérature en ancien français, textes. Bern : Francke Verlag.

Henry, Albert (1953) Chrestomathie de la littérature en ancien français I; notes ; glossaire; table des noms propres. Bern : Francke Verlag.

von Troyes, Kristian (1966) Yvain. Tübingen : Max Niemeyer Verlag.

La Bible (Le Nouveau Testament), Darby bible (1859), source internet: http://saintebible. $\mathrm{com} / \mathrm{matthew} / 27-24 . \mathrm{htm}$.

La Bible (Le Nouveau Testament), Louis Segond Bible (1910), source internet : http:// saintebible.com/matthew/27-24.htm.

La Bible (Le Nouveau Testament), Martin Bible (1744), source internet : http://saintebible.com/matthew/27-24.htm.

Lacroix, Daniel/ Philippe Walter (1989) Tristan et Iseut. Québec : Librairie Générale Française.

Moignet, Gérard (1969) La chanson de Roland. Paris : Editions Bordas.

Plutarque (1802) Les vies des hommes illustres, (Antonius), traduit par Jacques Amyot. Paris : Imprimerie de Cussac. 
Rabelais (1936) Gargantua ; Pantagruel, (livre second, extraits). Paris : Classiques Larousse.

ViLlon, François (1992) Poésies. Paris : GF- Flammarion.

\section{RÉSUMÉ}

Un essai sur la personne en diachronie : comment la perte de la flexion verbale change le point de vue sur la personne

Dans le présent article, l'auteur essaie de montrer, dans une perspective diachronique, comment la disparition de la flexion verbale a influé sur l'emploi (excessif) des possessifs en français moderne. En partant du latin où l'emploi du possessif avec les notions relevant de la sphère personnelle était plutôt rare, voire exceptionnel, il suit, à travers l'évolution de la langue française, l'apparition du possessif dans les syntagmes relatifs aux parties du corps, aux vêtements et à la famille. Il montre qu'à un moment donné de l'histoire du français, l'emploi du possessif dans ces syntagmes coexistait avec l'emploi de l'article défini. L'auteur explique que cela a résulté de la perte (partielle) de la personne du système verbal.

Mots-clés : flexion verbale, personne, sphère personnelle, inaliénabilité, possessif, article

\section{ABSTRACT}

An Essay on the Person in Diachrony: how the Loss of Verbal Inflections changes the Point of View on the Person

In the present article the author, seeks to show how the loss of the verbal inflection influences, from the diachronic point of view, the use of the possessive in contemporary French. Starting with Latin, in which the use of the possessive with concepts from the personal sphere was rather rare, even exceptional, the present article follows, through the different periods of the French language, the appearance of the possessives in constructions involving body parts, clothes and the family. It is shown that, in one specific moment in the history of the French, there was simultaneous use of the possessive and the article for the concepts in question. The author justifies that phenomenon as the consequence of the (partial) loss of the person from the French verbal system.

Keywords: verbal flexion, person, personal sphere, inalienability, possessive, article

\section{POVZETEK}

Oseba z diahronega gledišča : kako izguba glagolskega pregibanja vpliva na dojemanje osebe

V pričujočem članku skuša avtor z diahronega stališča prikazati, kako je postopno izginjanje glagolskega pregibanja vplivalo na (pretirano) rabo svojilnih zaimkov v moderni francoščini. Raba 
izhaja iz latinščine, kjer je bila uporaba svojilnih zaimkov za označevanje pojmov, pripadajočih osebni sferi, precej redka ali celo izjemna, nadaljuje s pregledom razvoja francoščine in opiše, kako se svojilni zaimki vedno pogosteje pojavljajo v imenskih zvezah, ki se nanašajo na dele telesa, oblačila in družino. V članku pokaže, da je v določenih obdobjih francoščina dovoljevala uporabo obeh, tako svojilnih zaimkov kot določnega člena. Avtor pojav razloži kot (delno) izgubo osebe iz glagolskega sistema.

Ključne besede: glagolsko pregibanje, oseba, osebna sfera, svojilni zaimki, člen 